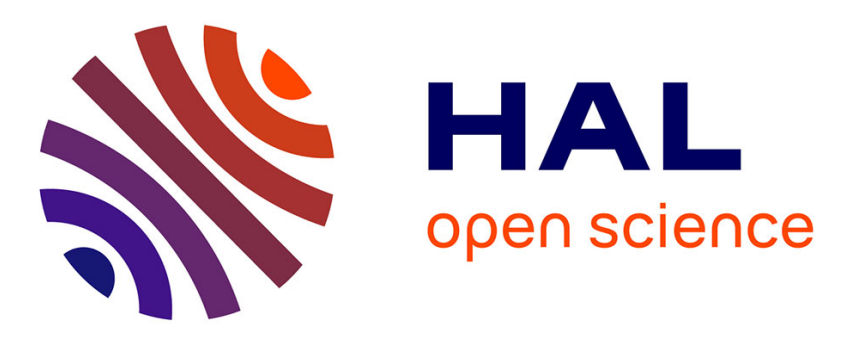

\title{
Brassicaceae INDEHISCENT genes specify valve margin cell fate and repress replum formation
}

\author{
Thomas Girin, Pauline Stephenson, Cassandra M P Goldsack, Sherry A \\ Kempin, Amandine Perez, Nuno Pires, Penelope A Sparrow, Thomas A \\ Wood, Martin F Yanofsky
}

\section{To cite this version:}

Thomas Girin, Pauline Stephenson, Cassandra M P Goldsack, Sherry A Kempin, Amandine Perez, et al.. Brassicaceae INDEHISCENT genes specify valve margin cell fate and repress replum formation. Plant Journal, 2010, 63 (2), pp.329-338. 10.1111/j.1365-313X.2010.04244.x . hal-01204205

\section{HAL Id: hal-01204205 \\ https://hal.science/hal-01204205}

Submitted on 30 May 2020

HAL is a multi-disciplinary open access archive for the deposit and dissemination of scientific research documents, whether they are published or not. The documents may come from teaching and research institutions in France or abroad, or from public or private research centers.
L'archive ouverte pluridisciplinaire HAL, est destinée au dépôt et à la diffusion de documents scientifiques de niveau recherche, publiés ou non, émanant des établissements d'enseignement et de recherche français ou étrangers, des laboratoires publics ou privés. 


\title{
Brassicaceae INDEHISCENT genes specify valve margin cell fate and repress replum formation
}

\author{
Thomas Girin ${ }^{1, \dagger}$, Pauline Stephenson ${ }^{1, \dagger}$, Cassandra M. P. Goldsack ${ }^{1}$, Sherry A. Kempin ${ }^{2}$, Amandine Perez ${ }^{1}$, Nuno Pires ${ }^{3}$, \\ Penelope A. Sparrow ${ }^{1}$, Thomas A. Wood ${ }^{1}$, Martin F. Yanofsky ${ }^{2}$ and Lars Østergaard ${ }^{1, *}$ \\ ${ }^{1}$ Department of Crop Genetics, John Innes Centre, Colney Lane, Norwich NR4 7UH, UK, \\ ${ }^{2}$ Section of Cell and Developmental Biology, University of California at San Diego, La Jolla, CA 92093-0116, USA, and \\ ${ }^{3}$ Department of Plant Sciences, University of Oxford, South Parks Road, Oxford OX1 3RB, UK
}

Received 12 February 2010; revised 9 April 2010; accepted 21 April 2010; published online 26 May 2010

*For correspondence (fax +44 1603 450027; e-mail lars.ostergaard@bbsrc.ac.uk).

${ }^{\dagger}$ These authors contributed equally to the work.

\begin{abstract}
SUMMARY
Members of the Brassicaceae family, including Arabidopsis thaliana and oilseed rape (Brassica napus), produce dry fruits that open upon maturity along a specialised tissue called the valve margin. Proper development of the valve margin in Arabidopsis is dependent on the INDEHISCENT (IND) gene, the role of which in genetic and hormonal regulation has been thoroughly characterised. Here we perform phylogenetic comparison of IND genes in Arabidopsis and Brassica to identify conserved regulatory sequences that are responsible for specific expression at the valve margin. In addition we have taken a comparative development approach to demonstrate that the BraA.IND.a and BoIC.IND.a genes from B. rapa and B. oleracea share identical function with Arabidopsis IND since ethyl methanesulphonate (EMS) mutant alleles and silenced transgenic lines have valve margin defects. Furthermore we show that the degree of these defects can be fine-tuned for crop improvement. Wild-type Arabidopsis produces an outer replum composed of about six cell files at the medial region of the fruits, whereas Brassica fruits lack this tissue. A strong loss-of-function braA.ind.a mutant gained outer replum tissue in addition to its defect in valve margin development. An enlargement of replum size was also observed in the Arabidopsis ind mutant suggesting a general role of Brassicaceae IND genes in preventing valve margin cells from adopting replum identity.
\end{abstract}

Keywords: Brassicaceae, INDEHISCENT, fruit development, tissue patterning, promoter element conservation, tissue specificity.

\section{INTRODUCTION}

During evolution, flowering plants have acquired a range of ways by which to effectively disperse their seeds and in this way optimise survival of the next generation. These mechanisms are often achieved by the formation of specific tissues that allow the fruit to efficiently release the seeds at maturity. Arabidopsis and Brassica species are members of the Brassicaceae family and produce inflorescences with cruciform flowers. Brassicaceae fruits develop as siliques in the centre of the flowers and elongate upon fertilisation to form a number of specialised tissues including the valves (or seed pod walls), a central replum and valve margins that form at the valve/replum borders where fruit opening will take place in a process known as dehiscence or pod shatter. This process is the result of highly coordinated processes, which allow the separation of the valves from the replum and lead to the timely dispersal of mature seeds (Roberts et al., 2002; Balanzá et al., 2006; Østergaard, 2009; Girin et al., 2009). Upon fruit maturation, the valve margins mediate fruit opening through degradation of a pectin-rich separation layer by secreted cell wall-degrading enzymes such as polygalacturonases (Petersen et al., 1996; Spence et al., 1996; Degan et al., 2001; Ogawa et al., 2009).

Setting up the overall patterning programme of the Brassicaceae fruit is a prerequisite for proper development and several of the key genetic factors from Arabidopsis have been identified (Dinneny and Yanofsky, 2005; AlonsoCantabrana et al., 2007) (Fig. 1). Replum tissue is specified by the REPLUMLESS (RPL) gene, which acts by limiting the 


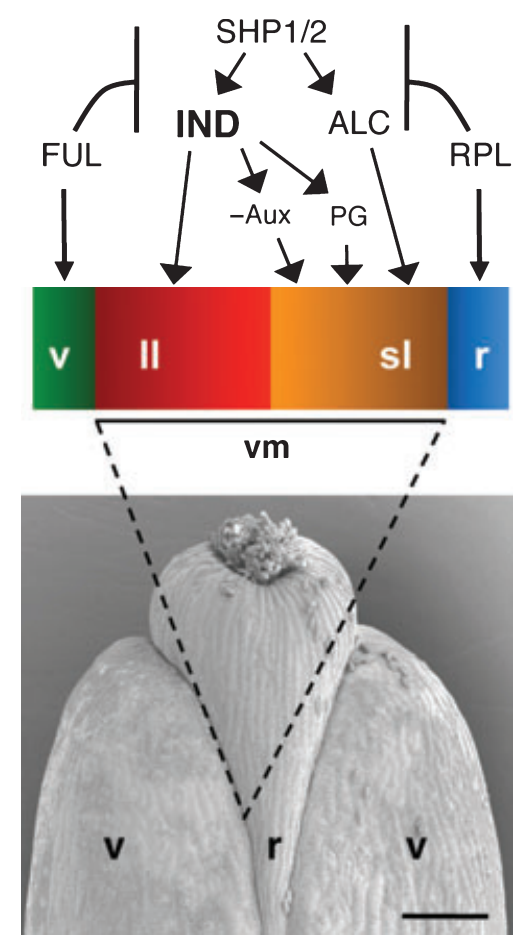

Figure 1. Overview of current knowledge on valve margin formation in Arabidopsis.

Top: Schematic outline of genetic and hormonal regulation of development of the lignified layer (red) and separation layer (orange). Valve and replum are indicated in green and blue, respectively.

Bottom: Scanning electron micrograph of the apical parts of a fruit from Arabidopsis thaliana ecotype Landsberg erecta. FUL, FRUITFULL; SHP1/2, SHATTERPROOF 1 and 2; RPL, REPLUMLESS; IND, INDEHISCENT; ALC, ALCATRAZ; PG, Polygalacturonase; -Aux, auxin minimum; v, valve; II, lignified layer; sl, separation layer; $r$, replum; vm, valve margin. Bar $=200 \mu \mathrm{m}$.

expression of valve margin identity genes SHATTERPROOF1 and -2 (SHP1/2), INDEHISCENT (IND) and ALCATRAZ (ALC) to the narrow stripes where valve margins will form (Liljegren et al., 2000, 2004; Roeder et al., 2003; Rajani and Sundaresan, 2001). In the valves, the FRUITFULL (FUL) gene is required for post-fertilisation elongation of the fruit $(\mathrm{Gu}$ et al., 1998) and acts similarly to RPL by repressing SHP1/2 and IND gene activity (Ferrándiz et al., 2000).

It was recently found that this genetic network controls distribution of the phytohormone auxin to ensure accurate fruit patterning (Sorefan et al., 2009). In wild-type fruits, local depletion of auxin at the valve margin is required for specification of the separation layer where fruit opening takes place. IND mediates the formation of this auxin minimum by regulating auxin transport (Sorefan et al., 2009).

Although efficient seed dispersal is an advantage for plants growing in the wild, asynchronous pod shatter presents a serious problem for oilseed rape production, with annual losses of $11-25 \%$ (Price et al., 1996). The elucidation of the genetic interactions during Arabidopsis fruit development has provided some clear directions on how to reduce asynchronous pod shatter in oilseed rape. We have previously shown in $B$. juncea that ectopic expression of the Arabidopsis FRUITFULL (FUL) gene under control of the Cauliflower Mosaic Virus 35S promoter results in completely pod shatter-resistant fruits similar to 35S::FUL transgenic Arabidopsis fruits (Østergaard et al., 2006; Ferrándiz et al., 2000). This result confirmed that knowledge gained on modulating traits from Arabidopsis fruit development can be successfully transferred to oilseed rape. Nevertheless, a total block of shattering is not desirable since this will lead to significant seed damage when opening the fruits, and it is clear that careful fine-tuning is required to optimise this trait.

Here we compare the function of IND genes identified in Brassica species with the IND gene of Arabidopsis (abbreviated AtIND hereafter). In addition to a universal role in promoting valve margin development, analysis of strong loss-of-function braA.ind.a and atind mutants revealed a function for Brassicaceae IND genes in repressing replum formation. We also demonstrate that the activity of these genes can be modified to either completely or partially inhibit valve margin formation with potential for oilseed rape breeding, and we identify a region upstream in the promoter of Arabidopsis and Brassica genes that is sufficient for valve margin-specific expression of the IND genes in the fruit.

\section{RESULTS}

\section{Diploid Brassica genomes contain one copy of a putative INDEHISCENT orthologous gene}

Based on synteny between Arabidopsis and B. rapa genomes, we identified 12 bacterial artificial chromosome (BAC) clones from B. rapa ssp. pekinensis (variety Chiifu) that were likely to contain a potential orthologue of the AtIND gene. Presence of an AtIND homologous gene was confirmed in all the clones by PCR, and sequencing of several independent PCR products revealed only one copy of the gene. Furthermore, only this gene was identified by sequencing of PCR products obtained with degenerate primers on genomic DNA and cDNA. This sequence was subsequently used to design primers for direct $B A C$ sequencing to obtain the entire gene sequence including the predicted open reading frame (ORF) of $516 \mathrm{bp}, 3110 \mathrm{bp}$ of promoter sequence and $1345 \mathrm{bp}$ of sequence downstream of the predicted stop codon (Fig. 2a). The gene was named BraA.IND.a in accordance with the standardised Brassica gene nomenclature ( $\varnothing$ stergaard and King, 2008) and the sequence has been deposited in GenBank (accession number GU386357). The $B$. rapa genome is called the $A$ genome and consists of 10 chromosomes (A1-A10). Based on the mapping of $\mathrm{BAC}$ clones, we found that the BraA.IND.a gene is located on $B$. rapa chromosome $A 3$ corresponding to the 
(a)

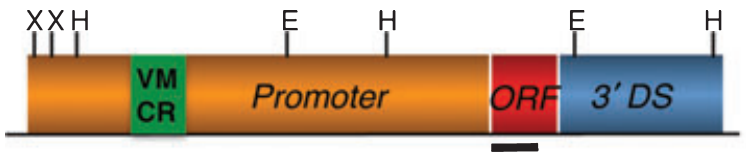

(b)
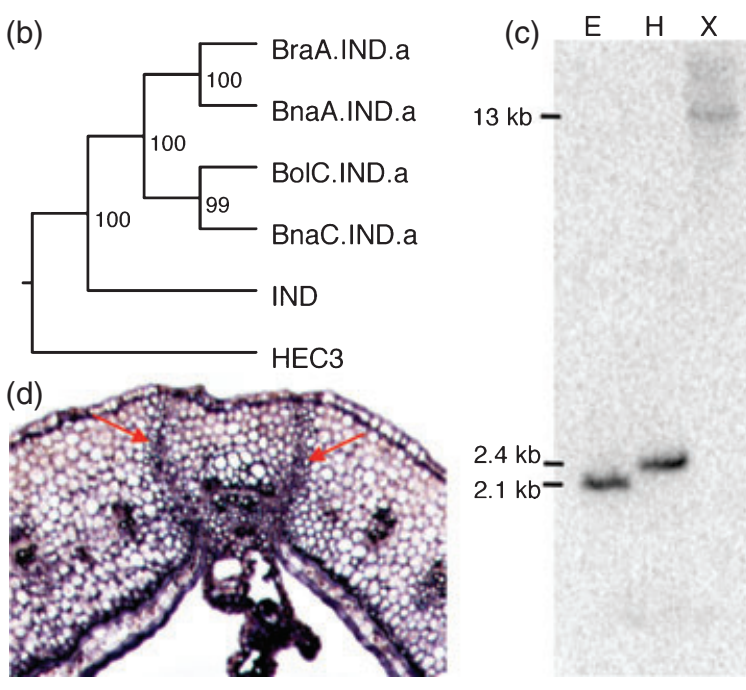

$2.4 \mathrm{~kb}$ $2.1 \mathrm{~kb}^{-}$

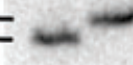

Figure 2. Sequence, copy number and expression analysis of Brassica and Arabidopsis IND genes.

(a) Gene structure of AtIND and BraA.IND.a. The promoter region is shown in orange, the open reading frame (ORF) is in red and the $3^{\prime}$ downstream sequence ( $3^{\prime}$ DS) of the gene is indicated in blue. The green region in the promoter, annotated VMCR (valve margin cis region), indicates the conserved region between Arabidopsis and Brassica IND genes containing cis regulatory sequences. The line below the ORF indicates the position of the probe used for the Southern blot in (c) and in situ hybridisation in (d).

(b) Phylogenetic tree showing the sequence relationship between Brassica and Arabidopsis IND ORFs. The IND-related Arabidopsis HECATE3 (HEC3) gene (Gremski et al., 2007) was used as an outgroup.

(c) Southern blot analysis using gDNA from B. rapa variety R-o-18. Three restriction enzymes were used $(\mathrm{E}, \mathrm{EcoRl} ; \mathrm{H}$, Hindlll; $\mathrm{X}, \mathrm{Xbal})$. Positions of restriction sites for these enzymes are shown in (a).

(d) In situ hybridisation showing expression at the valve margin of a $B$. rapa Ro-18 gynoecium at fertilisation (stage 12/13).

syntenic region of Arabidopsis chromosome IV where AtIND resides (Parkin et al., 2005). As for AtIND, the ORF of the $B$. rapa homologue is predicted to be contained within a single exon (Fig. 2a). The encoded BraA.IND.a protein shares $98 \%$ amino acid identity with the AtIND protein in the DNA-binding bHLH domain and $66 \%$ overall (Fig. S2 in Supporting Information).

After divergence of the Arabidopsis and Brassica genera about 20 million years ago (Yang et al., 1999; Koch et al., 2000; Parkin et al., 2005), the diploid Brassica genome underwent a triplication event (Lysak et al., 2005). Consequently, it is often the case that single-copy Arabidopsis genes exist as three paralogues in diploid Brassica genomes. To determine the number of BraA.IND genes, we performed a Southern blot analysis on genomic DNA from $B$. rapa using three restriction enzymes. Only one band

was observed in each lane at the expected size, which suggests that BraA.IND.a is present as a single-copy gene in the $B$. rapa genome (Fig. 2c). This observation is in agreement with the sequencing data, and it therefore suggests that $B$. rapa has lost two copies of this gene since the genome triplication event.

To ascertain whether this apparent gene loss is conserved among other members of the Brassica genus, we performed PCR with conserved oligos and isolated a sequence from $B$. oleracea genomic DNA with a high level of identity to the ORFs of AtIND (77\%) and BraA.IND.a (95\%). This gene was named BoIC.IND.a (GenBank accession number GU386356), and similar to the situation in $B$. rapa, only a single sequence was identified in $B$. oleracea when the PCR products were sequenced.

$B$. napus (oilseed rape) was formed by hybridisation between $B$. rapa and $B$. oleracea. As a consequence $B$. napus is allotetraploid consisting of two genomes; the $A$ genome from $B$. rapa and the $C$ genome from $B$. oleracea (U. N., 1935). In the GenBank database, we identified two putative IND sequences from $B$. napus (HB416515 and HB416517). A phylogenetic analysis revealed that instead of being most closely related to each other, the two $B$. napus IND-like sequences cluster with BraA.IND.a and BoIC.IND.a (Figs 2b and S2). This analysis furthermore shows that the Brassica IND genes identified here more closely resemble AtIND than the closest Arabidopsis homologue, HECATE3 (HEC3) (Pires and Dolan, 2009).

We subsequently mapped the chromosomal location of the two $B$. napus IND homologues in the Tapidor/Ningyou7 doubled haploid mapping population (Qiu et al., 2006). This analysis showed that the gene most closely related to BraA.IND.a is positioned on $B$. napus chromosome $A 3$ (HB416515, named BnaA.IND.a) whilst the one most closely related to BoIC.IND.a is positioned on B. napus chromosome C3 (HB416517, named BnaC.IND.a). Together, these results demonstrate that the two IND sequences identified in $B$. napus originate from each of the two diploid progenitors (B. rapa and $B$. oleracea).

\section{Conserved upstream sequence in the IND promoter is sufficient for valve margin expression and regulation by SHP and FUL genes}

In Arabidopsis, AtIND is required for valve margin formation and both in situ hybridisation and reporter lines have demonstrated that AtIND is indeed expressed in this tissue (Liljegren et al., 2004; Sorefan et al., 2009). In accordance with this, we performed in situ hybridisation on $B$. rapa fruits and found that the BraA.IND.a gene was expressed in valve margin cells (Fig. 2d). This result suggests that the function of the Arabidopsis and Brassica IND genes is conserved.

By aligning the promoter regions of AtIND with BraA.IND.a, we identified a 400-bp region about 1.8-2.2 kb upstream of the start codon with $86 \%$ sequence identity (Fig. S3). This is 


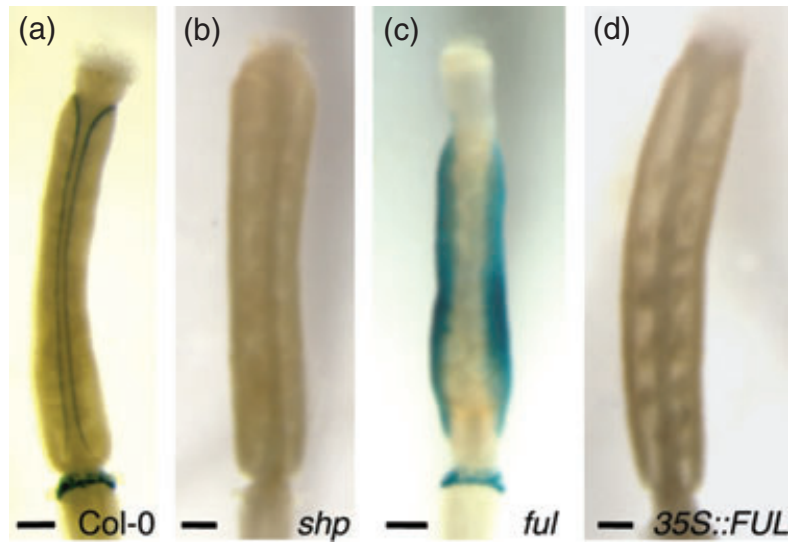

Figure 3. A 400-bp fragment of the AtIND promoter is sufficient for valve margin expression.

p400-m35S::GUS expression in wild-type Col-0 (a), shp 1/2 double mutant (b), ful-2 mutant (c) and 35S::FUL transgenic line (d). Bar $=500 \mu \mathrm{m}$.

remarkably high for a non-coding region and suggests either that it in fact does encode transcribed sequence or that it harbours regulatory sequences that are responsible for its expression pattern. By northern blot analysis using RNA from Arabidopsis fruit we were unable to detect any signal indicative of expression of upstream regions including the 400-bp sequence, although a clear signal was observed when using a probe in the ORF (Fig. S4).

From Arabidopsis genomic DNA, we cloned the 400-bp region in front of a minimal $35 \mathrm{~S}$ promoter driving the GUS reporter gene in the pDW294 vector (Busch et al., 1999). This construct, p400-m35S::GUS, was transformed into Arabidopsis and 28 out of 37 lines showed expression in the valve margin region (Fig. 3a) whereas the m35S::GUS alone did not (pDW294 in Fig. S5a). Staining in nectaries was occasionally observed, but this was also the case for the pDW294 without any IND promoter sequence (Fig. S5a).

Expression of AtIND at the valve margin was previously demonstrated to depend on the SHATTERPROOF1 and SHATTERPROOF2 (SHP1/2) genes, whereas AtIND expression in the valves is prevented by the activity of the FRUITFULL (FUL) gene (Liljegren et al., 2000; Ferrándiz et al., 2000). These data were obtained with an enhancer trap line, GT140, in which the GUS reporter is inserted $3 \mathrm{~kb}$ upstream of the AtIND gene (Liljegren et al., 2000). Crosses of the p400-m35S::GUS line to the shp1/2 double mutant, ful single mutant and the 35S::FUL transgenic line revealed that regulation of this construct is indistinguishable from the regulation of GT140 expression (Fig. 3b-d). These results confirm that regulation of AtIND expression by SHP1/2 and FUL occurs via elements in this 400-bp region.

Positioned close to the centre of the 400 -bp region is a CArG box element, which is conserved among the Arabidopsis and Brassica IND genes (Fig. S3). CArG boxes are binding sites for the transcription factor family of MADS box proteins (Riechmann et al., 1996). Since both SHP1/2 and FUL belong to this family, we speculated whether this sequence is required for the valve margin expression of AtIND. To this end we mutated the CArG box sequence, but found that it had no effect on the valve margin specificity (Fig. S5d). Moreover, a 20-bp probe containing this element was not bound by either SHP1/2 or FUL protein in an in vitro gelshift assay (data not shown).

Overlapping fragments containing the $5^{\prime}$ half or the $3^{\prime}$ half of the 400-bp region in front of $\mathrm{m} 35 \mathrm{~S}$ ::GUS showed no valve margin expression (Fig. S5b and c) suggesting that interactions between factors that bind different elements in the 400-bp region are involved in regulating tissue-specificity.

\section{Brassica IND genes function to specify valve margin and repress replum formation}

Similarities in sequences and expression patterns of genes from different species are good indications that they have similar function. However, the strongest evidence for such conservation comes from analysis of loss-of-function mutants. We used the recently developed reverse genetics platform for TILLING (targeting induced local lesions in genomes) in B. rapa variety R-o-18 (Stephenson et al., 2010) to identify a number of lines with point mutations in the BraA.IND.a gene. Three alleles were selected for further analysis and grown to homozygosity. The braA.ind.a-1 allele has a premature stop codon at the beginning of the bHLH domain $(\mathrm{Q} 102 \mathrm{X})$ expected to completely disrupt the function of the gene. braA.ind.a-2 has a mis-sense mutation in the IND-specific $\mathrm{N}$-terminal region changing serine to phenylalanine (S37F), while braA.ind.a-3 has a mis-sense mutation in a region sharing homology with the HEC genes (Gremski et al., 2007) that leads to a change of glutamic acid to lysine (E82K).

Homozygous braA.ind.a mutants showed a dramatic loss of valve margin formation compared with wild-type R-o-18 (Fig. 4a-f), which was visible both by scanning electron microscopy (SEM) and tissue-staining of cross sections. It was not possible to identify a valve margin defect in the two mis-sense mutant alleles, braA.ind.a-2 and braA.ind.a-3, by SEM (Fig. 4g,j); however, close examination of the valve margin region in cross sections revealed a lack of small cells that are characteristic of this tissue, thus suggesting that both of these alleles have partially lost valve margin identity (Fig. 4h,i,k,l).

To test if the BoIC.IND.a gene has the same function as BraA.IND.a and AtIND, we adopted an RNA silencing approach. The BoIC.IND.a ORF was cloned as inverted tandems with an intron sequence in between and expressed behind the $35 \mathrm{~S}$ promoter. This construct was transformed into B. oleracea genotype DH1012 by Agrobacterium tum efaciens-mediated transformation and transgenic shoot regeneration. Out of 13 primary transgenic plants, seven exhibited a strong defect in fruit opening compared with 
Figure 4. Mutations in the Brassica IND gene lead to defects in valve margin formation. Analysis of wild-type B. rapa var. R-o-18 (a-c), braA.ind.a-1 (d-f), braA.ind.a-2 (g-i) and braA.ind.a-3(j-I) mutant alleles at developmental stage 17A by scanning electron microscopy (a, $d$, $\mathrm{g}, \mathrm{j})$ and Alcian Blue/Saffranin O-staining of cross sections (b, $c, e, f, h, i, k, I)$. (m)-(o) Cross sections of stage-17C fruits from $B$. oleracea genotype DH1012 untransformed $(\mathrm{m})$ and from two independent lines transformed with a BoIC.IND.a silencing construct $(n, 0)$ stained by Alcian Blue/ Saffranin O. Bars $=500 \mu \mathrm{m}(\mathrm{a}, \mathrm{d}, \mathrm{g}, \mathrm{j}), 100 \mu \mathrm{m}(\mathrm{b}$, e, h, k, m, n, o), $20 \mu \mathrm{m}(\mathrm{c}, \mathrm{f}, \mathrm{i}, \mathrm{l})$.
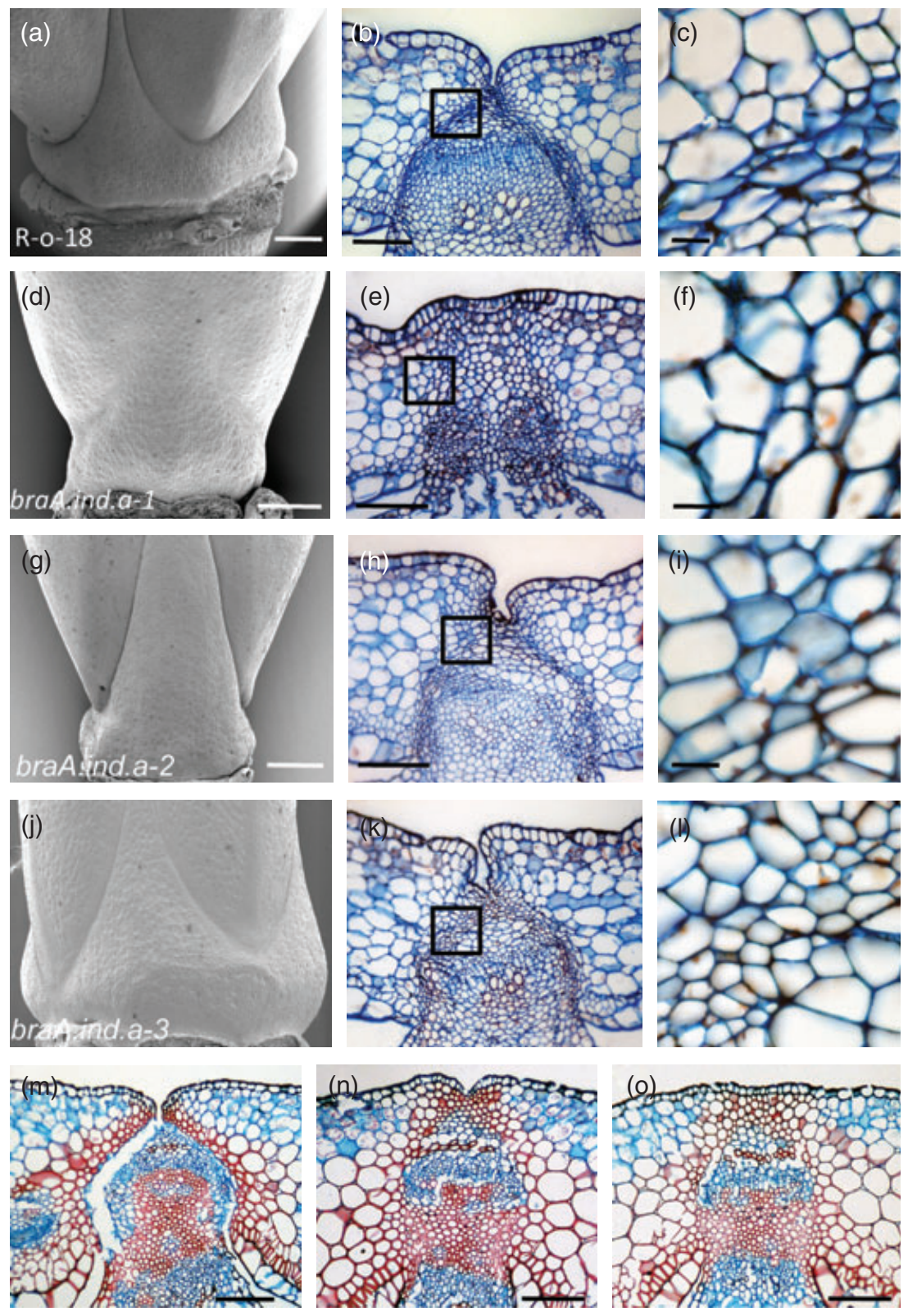

both the untransformed tissue culture-regenerated controls and wild-type $B$. oleracea DH1012 fruits. The identical phenotype was observed in progeny of the primary transgenic lines at a 3:1 ratio. We characterised two transgenic lines in more detail by tissue staining of cross sections, and found that fruits from these lines had lost the ability to form valve margin tissue (Fig. 4m-0). BoIC.IND.a RNA levels were indeed significantly reduced in these lines (Fig. S6), and the results therefore demonstrate that IND gene function is conserved between members of the Brassica genus.

When analysing the strong braA.ind.a-1 mutant allele, we observed that in addition to valve margin defects these fruits had gained an outer replum, which is not observed in the wild type (Fig. $5 a, b, e, f$ ) or in the weaker mutant alleles. A similar gain-of-replum effect was observed in one of the two BoIC.IND.a RNA interference (RNAi)i lines compared with untransformed DH1012 (Fig. $4 \mathrm{~m}$ and o). This observation shows that Brassica IND genes are repressors of replum formation, and to test if a similar function could be revealed for AtIND in Arabidopsis, we analysed replum morphology of ind versus wild-type Arabidopsis fruits. SEM photographs and measurements of replum width and counting number of cell files (Figs $5 c, d$ and $S 7$ ) revealed a significant enlargement of replum tissue with additional cell files in the ind mutant background. Together these results demonstrate that IND genes from Brassica and Arabidopsis function to promote valve margin development and repress replum formation. 

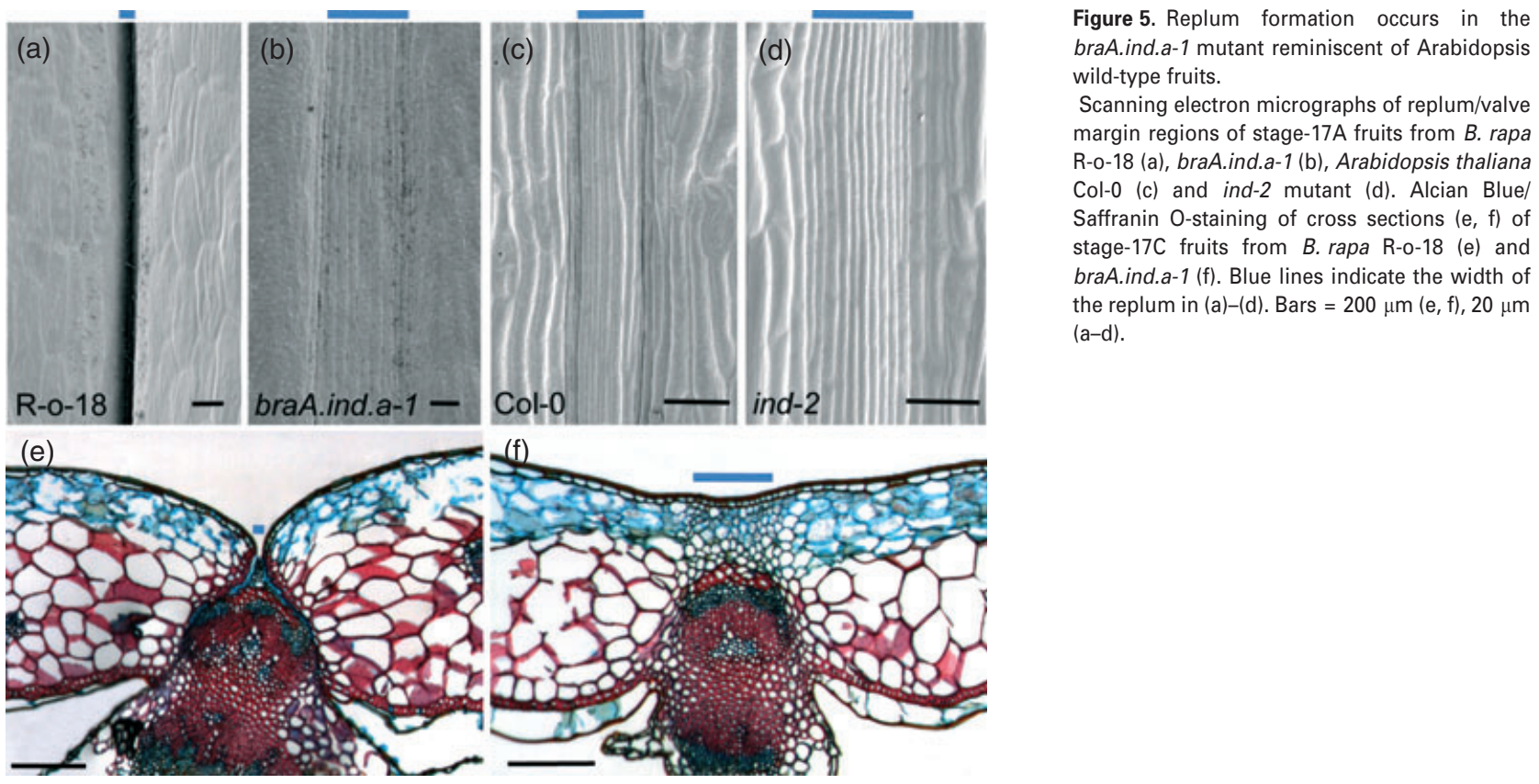

\section{DISCUSSION}

Fruits from members of the Brassicaceae encapsulate and nurture the seeds from fertilisation until maturation. At maturity, Brassicaceae fruits open along a specific tissue called the valve margin, allowing the separation of the valves from the replum. This dehiscence process is carefully controlled so that opening of the fruit will only take place when the seeds are fully developed. In Arabidopsis, it has previously been demonstrated that the bHLH transcription factor IND is required for valve margin formation (Liljegren et al., 2004; Sorefan et al., 2009), and here we show that IND orthologous genes in members of the Brassica genus also have this function. We also show an additional function of IND from both Arabidopsis and $B$. rapa in repressing the formation of an adjacent tissue, the replum.

The BraA.IND.a gene in B. rapa was completely sequenced including the single-exon ORF, more than $3 \mathrm{~kb}$ of sequence upstream of the predicted start codon and around $1.3 \mathrm{~kb}$ downstream of the predicted stop codon. Expression of BraA.IND.a was found at the valve margin of $B$. rapa fruits, in agreement with a role in specifying this tissue.

Southern blot analysis indicated that BraA.IND.a exists as a single-copy gene in B. rapa. Since the diploid Brassica genome underwent a triplication event after diverging from the common ancestor of Arabidopsis and Brassica, it is most likely that the Brassica diploid genome has lost two copies of the gene. Previous work has shown the dramatic developmental effects that ectopic expression of AtIND has in Arabidopsis (Sorefan et al., 2009). Perhaps accumulation of mutations leading to mis-expression of BraA.IND genes provided a substantial disadvantage for such individuals and was therefore selected against by inactivation/deletion of the genes. In contrast, a high selective pressure on the expression pattern of the remaining gene may have ensured its specific activity at the valve margin.

Brassica napus (oilseed rape) originates from a cross between the diploid progenitors $B$. rapa and $B$. oleracea (U. N., 1935) which is likely to have taken place during human cultivation approximately 10000 years ago. A phylogenetic analysis of Brassica IND cDNA sequences suggests that each of the two diploid genomes in $B$. napus contains one IND orthologue. This is consistent with Brassica IND genes existing as single copies in diploid Brassica species. Since the chromosomal location of the two Brassica IND genes identified here occur in equivalent regions of the respective genomes (chromosomes $\mathrm{A} 3$ and $\mathrm{C} 3$ ), the data suggest that Brassica IND gene loss may have happened soon after the genome triplication event and prior to diversification of $B$. rapa and $B$. oleracea about 3.7 million years ago (Inaba and Nishio, 2002).

We identified a 400-bp region in the promoters of Arabidopsis and Brassica IND genes that is highly conserved and sufficient for valve margin expression and regulation by factors that control AtIND expression in Arabidopsis (Fig. 3). Our results suggest that there may be at least two interacting cis-regulatory elements within this 400-bp sequence, both necessary for valve margin expression of the gene. It is likely that the valve margin-specific expression of IND genes is particularly sensitive to mutations in this region, as sug gested by the high level of sequence conservation between Brassicaceae genera.

Since the IND gene is regulated by MADS box transcription factors (Liljegren et al., 2000; Ferrándiz et al., 2000), it 
was intriguing that a MADS box recognition motif (CArG box) was identified in this region. However, reporter gene analysis showed that this sequence is not required for valve margin expression. A scan of the region for other known cis-regulatory sequences using the PLACE database (Higo et al., 1999) did not reveal the presence of any obvious candidate elements, except for a non-canonical CArG box, which is, however, not conserved in B. rapa. Hence additional studies will be required to elucidate the sequences involved and the factors binding to them.

The function of the BraA.IND.a gene was unequivocally established by the identification of loss-of-function mutations in this gene in B. rapa. Fruits from the strong braA.ind.a-1 mutant allele had completely lost valve margin formation, whereas weaker alleles had partial defects (Fig. 4). Likewise, silencing of the BolC.IND.a gene by RNA silencing also led to fruits with strong defects in valve margin specification.

Fruits from the Brassicaceae family produce a replum at the medial plane of the fruit that connects to the valves via the valve margin tissue. At the interior the replum contains a large vascular bundle, and at the exterior Arabidopsis fruits produce an outer replum of approximately six files of highly elongated cells that are narrower than valve cells (Fig. 5c). This is in contrast to fruits from Brassica species that have little or no outer replum cell files (Fig. 5). However, in the strong braA.ind.a-1 mutant allele and in a BoIC.IND.a RNAi line, we observed formation of outer replum tissue in addition to the defect in valve margin development, suggesting that BraA.IND.a is a repressor of replum formation. This observation was supported by the development of enlarged repla with additional cell files in an Arabidopsis ind mutant compared with wild type, and our data therefore show that IND genes from Brassicaceae are repressors of replum tissue formation. It may be possible to expand this statement to valve margin identity genes in general, since mutations in the SHP genes rescues the replum defect of a $\mathrm{rpl}$ mutant in Arabidopsis (Roeder et al., 2003). The absence of replum tissue in the braA.ind.a-2 and braA.ind.a-3 mutants as well as in a B. oleracea RNAi line suggests that valve margin development is more sensitive to a reduction in IND activity than repression of replum cell identity.

Control of fruit dehiscence (or pod shatter) is of considerable agronomic importance as oilseed rape farmers loose $11-25 \%$ of their harvest each year due to asynchronous fruit opening and seeds falling to the ground in the field (Price et al., 1996). We have previously shown that fruit-opening in $B$. juncea can be prevented by expressing the Arabidopsis FUL gene under control of the 35S promoter (Østergaard et al., 2006). Although completely sealed Brassica fruits such as the 35S::FUL plants and the braA.ind.a-1 mutant allele presented here are unsuitable for agronomic purposes, our data show that partial dehiscence can be achieved by fine-tuning the level of BraA.IND.a activity. This finding may have important implications for future strategies for control of pod shatter either by introgression of the alleles presented here or by screening for similar mutations in existing B. napus TILLING populations (Wang et al., 2008).

Over the last few decades, work in the model plant Arabidopsis has led to astounding leaps in our understanding of plant growth and development, and has in some instances pointed out clear directions for crop improvement, particularly in the closely related Brassica crop species. The results presented here demonstrate that the direction of this flow of information may be reversed, and that work in Brassica can reveal fundamental aspects of plant development which are unlikely to have been obtained by restricting the studies to Arabidopsis.

\section{EXPERIMENTAL PROCEDURES}

\section{Plant material and growth conditions}

Arabidopsis thaliana ecotypes Columbia (Col-0) and Landsberg erecta, mutants and transgenics were grown in the glasshouse under long-day conditions ( $16 \mathrm{~h}$ light) at $21^{\circ} \mathrm{C}$. Developmental stages of Arabidopsis flowers and fruits were defined as in Smyth et al. (1990).

Seeds of $B$. rapa var. R-o-18 wild type (Rusholme et al., 2007) and braA.ind.a mutant alleles were sown in John Innes No. 2 compost and kept at $7{ }^{\circ} \mathrm{C}$ with no light for 3 days before being transferred into the glasshouse at $18^{\circ} \mathrm{C}$ with $16 \mathrm{~h}$ light. The braA.ind.a mutants were obtained by TILLING in a B. rapa population described in Stephenson et al. (2010). Developmental stages for B. rapa flower and fruit developmental stages were defined based on Smyth et al. (1990), but slightly modified with the division of stage 14 into $14 \mathrm{~A}$ and $14 \mathrm{~B}$ and stage 17 into stage 17A, 17B and 17C (Fig. S1).

\section{Identification of BACs containing the BraA.IND.a gene and verification by direct BAC DNA sequencing}

A database of BAC end sequences from B. rapa ssp. Pekinensis (variety Chiifu) was searched at http://atidb.org using the accession number for the AtIND gene (At4g00120) to identify BAC clones that, based on alignment of their end sequences on the Arabidopsis genome, may contain potential orthologues of AtIND. Some of the $\mathrm{BAC}$ end sequences were highly similar to the AtIND sequence, and were used in combination with the Arabidopsis sequence and two putative $B$. napus IND sequences available in the GenBank database (HB416515 and HB416517) to design primers in conserved regions of the gene. These primers were then used to obtain the whole BnaA.IND. a sequence by sequencing directly, using both BAC DNA as template and PCR products obtained from BAC clones, genomic DNA and cDNA from B. rapa.

\section{BnaA.IND.a and BnaC.IND.a mapping in a Tapidor/Ningou7 population}

BnaA.IND.a and BnaC.IND.a were mapped in the Tapidor/Ningyou 7, doubled haploid, $B$. napus population using single-strand conformation polymorphism (SSCP) analysis according to Bertin et al. (2005). Briefly, PCR products were generated using primers BIND-F (5'-TTGCCAACGTTGAGGTAGCT-3') and BIND-R (5'-CGCTGTGTCATTATCTGACC-3') and denatured immediately prior to resolution in 
a non-denaturing gel system; $0.5 \times \mathrm{MDE}$ gel solution, $0.6 \times \mathrm{TBE}$ (Lonza Rockland, http://www.lonza.com/). After electrophoresis, bands were visualised by silver staining as described in Bassam et al. (1991). Genetic linkage mapping was performed using JoINMAP V.3.0 software (Stam, 1993).

\section{Generation of GUS reporter lines}

AtIND promoter fragments were cloned into the Hindlll restriction site of the pDW294 plasmid (Busch et al., 1999), thereby placing these fragments in front of a minimal Cauliflower Mosaic Virus 35S promoter driving expression of the $\beta$-glucoronidase gene (GUS). In p400-m35S::GUS the fragment was generated using primers IND125 (5'-AAGCTTTGGGCAATACCTTAATGTCG-3') and IND126 (5'-AAGCTTGCCATAAGGAAGCATAAGTCG-3'); in p5'VMCR primers IND125 and IND124 (5'-AAGCTTCTAAACCCCACGGTTTCTTCG-3') were used whereas primers IND117 (5'-AAGCTTGTAAGAAAATGTCGAAG-3') and IND116 (5'-AAGCTTTTTCGCTAACAAATTTC- $3^{\prime}$ ) were used to generate the fragment for the $\mathrm{p} 3^{\prime} \mathrm{VMCR}$ construct. The $\mathrm{p} \triangle \mathrm{CArG}$ construct was created by overlapping PCR, first generating two fragments using primers IND19 $\left(5^{\prime}\right.$-AAAAGGCC TTATGTTAATATCACCGTAGG- $\left.3^{\prime}\right)$ and INDNCO2 (5'-CCATGGCAAT TTAACATCATTGTTCTTC-3') together and INDNCO1 (5'-CCATGGTG TATAGGAGTTGTCAAAAG-3') and IND110 (5'-AAAACCGGTGGGTT GGGAGTTGTGG-3') together. The entire fragment without the CArGbox was then generated using primers IND19 and IND116 (5'-AAGCTTTTTCGCTAACAAATTTC- $3^{\prime}$ ) in the presence of the two partially overlapping fragments and subsequently cloned into to the Hindlll site of pDW294 in the sense orientation with respect to the minimal $35 \mathrm{~S}$ promoter. Transgenic plants were selected by kanamycin resistance after $A$. tumefaciens-mediated transformation into Arabidopsis (Col-0 ecotype).

\section{Construct for BoIC.IND.a silencing in B. oleracea}

A DNA fragment containing the entire ORF of BolC.IND.a was amplified using primers BIND3 (5'-AAAAAGCAGGCTATGTCTGGCT CAAAAGCAGATGC-3') and BIND4 (5'-AGAAAGCTGGGTTCAGACA TAGGAGCTCCAAGC-3'). The resulting fragment was Gateway cloned into the pBRACT507 vector (http://www.bract.org/constructsavailable/pBractVectors/Constructs/constructs.html) to generate the pRNAi-IND plasmid. pBRACT507 is designed for RNA silencing, containing the inserts as inverted repeats with a short intron in between and expression driven by the $35 \mathrm{~S}$ promoter.

\section{Brassica oleracea transformation}

A genetically uniform doubled haploid $B$. oleracea genotype $\mathrm{DH}$ 1012 (Sparrow et al., 2004) was transformed using the A. tumefaciens strain AGL1 (Lazo et al., 1991) harbouring the pRNAi-IND plasmid. The transformation protocol was as described in Sparrow et al. (2006) and on the BRACT website (http://www.bract.org) with the following modifications: Explants were initially selected on a low kanamycin concentration $(5 \mathrm{mg} / \mathrm{L}$ ) to compensate for use of the weaker NOS promoter driving the nptll gene. Regenerated shoots were later isolated and maintained on $25 \mathrm{mg} / \mathrm{L}$ kanamycin. Several lines exhibited strong fruit opening defects, and four lines were chosen for phenotypic analysis of hemizygous primary transgenics and of homozygous individuals in the following generation.

\section{Quantitative RT-PCR}

RNA extraction and reverse transcription (RT) reaction from stage12 gynoecia were performed as described in Girin et al. (2007). The quantitative (Q)-PCR reaction was carried out using SYBR Green JumpStart Taq ReadyMix (Sigma, http://www.sigmaaldrich.com/) in a Chromo4 thermocycler (Bio-Rad, http://www.bio-rad.com/).
Specific primers used were: 5'-AACCGCCGTAACGTAAGGGTAAG-3' and 5'-TGTGGTAATAACAAAGGCGAGAAGG-3' for BolC.IND.a and $5^{\prime}$-TTCAATGTCCCTGCCATGTA- ${ }^{\prime}$ and $5^{\prime}$-GAGACGGAGGATAG CGTGAG-3' for the internal control ACT2/7 (Marmagne et al., 2010). Relative mRNA accumulation was calculated using the standard curve method (Rutledge and Côté, 2003), with all amplification efficiencies being above 1.8. Values in Fig. $\mathrm{S} 6$ correspond to the average of three biological repeats \pm SD.

\section{GUS staining}

Fruits were pre-fixed in $90 \%$ acetone $\left(4^{\circ} \mathrm{C}\right)$ for 20 min at room temperature. Samples were subsequently rinsed with the reaction buffer [50 $\mathrm{mm} \mathrm{Na}_{2} \mathrm{PO}_{4}$ (pH 7.2), $0.2 \%$ Triton X-100, 5 mm ferrocyanide, $5 \mathrm{~mm}$ ferricyanide) and incubated overnight in reaction buffer with $2 \mathrm{~mm} \mathrm{X-Gluc} \mathrm{at} 37^{\circ} \mathrm{C}$. To clear the samples, a series of ethanol washes was performed (50-95\% in multiple steps) after incubation. GUS-stained tissue was examined under light microscopy.

\section{Alcian blue/Saffranin-0 staining}

Tissues were fixed for $6 \mathrm{~h}$ in an FAA solution $(3.7 \%$ formaldehyde, $5 \%$ acetic acid, $50 \%$ ethanol) and subsequently dehydrated through an ethanol series. The tissues were cleared with Histoclear (National Diagnostics, http://www.nationaldiagnostics.com/) and embedded in paraffin. An RM2255 rotary microtome (Leica, http://www. leica.com//) was used to make $8 \mu \mathrm{m}$ transverse stem sections. After deparaffinisation, sections were stained with an Alcian Blue $8 \mathrm{Gx}$ Safranin-O solution [0.05\% Alcian Blue $8 \mathrm{Gx}$ and $0.01 \%$ Safranin-O in $0.1 \mathrm{M}$ acetate buffer $(\mathrm{pH} 5.0)]$ as described in Østergaard et al. (2006). Sections were examined under light microscopy.

\section{Light microscopy}

Light microscopy images were obtained using a MZ16 stereomicroscope (Leica) and the images were captured with a DFC280 digital camera (Leica). Measurements were performed on captured images using Leica Application Suite (LAS) software.

\section{Scanning electron microscopy}

Fruit were fixed for approximately $6 \mathrm{~h}$ at $25^{\circ} \mathrm{C}$ in FAA and subsequently dehydrated through an ethanol series. After critical point drying, tissues were coated with gold and examined in a Philips XL30 FEG microscope using an acceleration voltage of $3 \mathrm{kV}$.

\section{Southern blot}

Ten micrograms of genomic DNA from R-o-18 was completely digested with each restriction enzyme indicated in Fig. 2c and run on a $0.5 \%$ agarose gel. The Southern blotting protocol was as in Sambrook and Russell (2001). A ${ }^{32}$ P-labelled probe was generated using a fragment of the BraA.IND.a ORF (positions 138-538) and the Random Prime Labelling System from Amersham Biosciences (http://www.gelifesciences.com). After hybridisation with this probe overnight at $65^{\circ} \mathrm{C}$, the filter was washed in $0.2 \times \mathrm{SSC}$ at $65^{\circ} \mathrm{C}$ and exposed to a Storage Phosphor screen (Molecular Dynamics) for 7 days before visualisation in a Typhoon 9200 phosphoimager (Amersham Biosciences, http://www.gelifesciences.com).

\section{Northern blot}

Five micrograms of total RNA from Arabidopsis fruits was loaded on $1.5 \%$ agarose formaldehyde gel. Northern blotting was performed as described by Sambrook and Russell (2001). Preparation of probes, hybridisation and subsequent washes was as described above for Southern blotting. 


\section{In situ hybridisation}

In situ hybridisation was performed essentially as described (Long et al., 2002) on tissue from B. rapa var. R-o-18 fruits at developmental stage 12. Tissue fixation, embedding and sectioning was carried out as described above. For hybridisation, an antisense ribonucleotide probe of BraA.IND.a was synthesised (positions 538138 ) by cloning the fragment in the pCR2.1 vector (Invitrogen, http:// www.invitrogen.com/) and selection for antisense orientation with respect to the $\mathrm{T7}$ promoter. The resulting plasmid was linearised by Spel digestion and the antisense probe was prepared using T7 RNA polymerase.

\section{Phylogenetic analysis}

The nucleotide sequences of ORFs were manually aligned. The most parsimonious tree was found using the DNAPENNY programme implemented in the PHYLIP package (Felsenstein, 1989) and bootstrapped 100 times. The tree was rooted with HEC3 (At5g09750).

\section{ACKNOWLEDGEMENTS}

We thank Kim Findlay at the John Innes Centre for assistance on SEM analysis and the John Innes Centre horticultural staff for their valuable and skilled help with general plant husbandry. We also wish to thank Nicolas Arnaud, George Averell and Sara Fuentes for discussions on the manuscript. This work was supported by grants BB/E006965/1 and BB/D018005/1 from the Biotechnology and Biological Sciences Research Council (BBSRC) to $L \varnothing$ and by a grant from the National Science Foundation to MFY.

\section{SUPPORTING INFORMATION}

Additional Supporting Information may be found in the online version of this article:

Figure S1. Developmental stages of B. rapa R-o-18 flowers and fruits.

Figure S2. CLUSTALW alignment of the Brassica and Arabidopsis IND and the Arabidopsis HEC3 amino acid sequences based on the open reading frame sequences used for the phylogeny shown in Figure $2 b$.

Figure S3. Alignment of the 400-bp promoter region of AtIND and BraA.IND.a.

Figure S4. Northern blot analysis to test for expression of regions in the upstream region of the IND gene.

Figure S5. GUS reporter staining of fruit from various lines.

Figure S6. Expression analysis of Bo/C.IND.a in stage-12 gynoecia. Figure S7. Replum from ind-2 mutant fruits are wider than wildtype fruits both in absolute width and in number of replum cell files.

Please note: As a service to our authors and readers, this journal provides supporting information supplied by the authors. Such materials are peer-reviewed and may be re-organized for online delivery, but are not copy-edited or typeset. Technical support issues arising from supporting information (other than missing files) should be addressed to the authors.

\section{REFERENCES}

Alonso-Cantabrana, H., Ripoll, J.J., Ochando, I., Vera, A., Ferrándiz, C. and Martínez-Laborda, A. (2007) Common regulatory networks in leaf and fruit patterning revealed by mutations in the Arabidopsis ASYMMETRIC LEAVES1 gene. Development, 134, 2663-2671.

Balanzá, V., Navarrete, M., Trigueros, M. and Ferrándiz, C. (2006) Patterning the female side of Arabidopsis: the importance of hormones. J. Exp. Bot $57,3457-3469$.
Bassam, B.J., Caetano-Anolles, G. and Gresshoff, P.M. (1991) Fast and sensitive silver staining of DNA in polyacrylamide gels. Anal. Biochem. 196, 80-83.

Bertin, I., Zhu, J.H. and Gale, M.D. (2005) SSCP-SNP in pearl millet - a new marker system for comparative genetics. Theor. Appl. Genet. 110, 14671472.

Busch, M.A., Bomblies, K. and Weigel, D. (1999) Activation of a floral homeotic gene in Arabidopsis. Science, 23, 585-587.

Degan, F.D., Child, R., Svendsen, I. and Ulvskov, P. (2001) The cleavable $\mathrm{N}$-terminal domain of plant endopolygalacturonases from clade $\mathrm{B}$ may be involved in a regulated secretion mechanism. J. Biol. Chem. 276, 3529735304.

Dinneny, J.R. and Yanofsky, M.F. (2005) Drawing lines and borders: how the dehiscent fruit of Arabidopsis is patterned. Bioessays, 27, 42-47.

Felsenstein, J. (1989) PHYLIP - Phylogeny Inference Package (Version 3.2). Cladistics, 5, 164-166.

Ferrándiz, C., Liljegren, S.J. and Yanofsky, M.F. (2000) Negative regulation of the SHATTERPROOF genes by FRUITFULL during Arabidopsis fruit development. Science, 289, 436-438.

Girin, T., Sorefan, K. and Østergaard, L. (2009) Meristematic sculpting in fruit development. J. Exp. Bot. 60, 1493-1502.

Girin, T., Lejay, L., Wirth, J., Widiez, T., Palenchar, P.M., Nazoa, P., Touraine, B., Gojon, A. and Lepetit, M. (2007) Identification of a $150 \mathrm{bp}$ cis-acting element of the AtNRT2.1 promoter involved in the regulation of gene expression by the $\mathrm{N}$ and $\mathrm{C}$ status of the plant. Plant Cell Environ. 30, 13661380.

Gremski, K., Ditta, G. and Yanofsky, M.F. (2007) The HECATE genes regulate female reproductive tract development in Arabidopsis thaliana. Development, 134, 3593-3601.

Gu, Q., Ferrándiz, C., Yanofsky, M.F. and Martienssen, R. (1998) The FRUITFULL MADS-box gene mediates cell differentiation during Arabidopsis fruit development. Development, 125, 1509-1517.

Higo, K., Ugawa, Y., Iwamoto, M. and Korenaga, T. (1999) Plant cis-acting regulatory DNA elements (PLACE) database:1999. Nucleic Acids Res. 27, 297-300.

Inaba, R. and Nishio, T. (2002) Phylogenetic analysis of Brassiceae based on the nucleotide sequences of the S-locus related gene, SLR1. Theor. Appl. Genet. 105, 1159-1165.

Koch, M.A., Haubold, B. and Mitchell-Olds, T. (2000) Comparative evolutionary analysis of chalcone synthase and alcohol dehydrogenase loci in Arabidopsis, Arabis, and related genera (Brassicaceae). Mol. Biol. Evol. 17, 1483-1498.

Lazo, G.R., Stein, P.A. and Ludwig, R.A. (1991) A DNA Transformation-Competent Arabidopsis Genomic Library in Agrobacterium. Biotechnology, 9, 963-967.

Liljegren, S.J., Ditta, G.S., Eshed, Y., Savidge, B., Bowman, J.L. and Yanofsky, M.F. (2000) SHATERPROOF MADS-box genes control seed dispersal in Arabidopsis. Nature, 404, 766-770.

Liljegren, S.J., Roeder, A.H., Kempin, S.A., Gremski, K., Østergaard, L., Guimil, S., Reyes, D.K. and Yanofsky, M.F. (2004) Control of fruit patterning in Arabidopsis by INDEHISCENT. Cell, 116, 843-853.

Long, J.A., Woody, S., Poethig, S., Meyerowitz, E.M. and Barton, M.K. (2002) Transformation of shoots into roots in Arabidopsis embryos mutant at the TOPLESS locus. Development, 129, 2797-2806.

Lysak, M.A., Koch, M.A., Pecinka, A. and Schubert, I. (2005) Chromosome triplication found across the tribe Brassiceae. Genome Res. 15, 516-525.

Marmagne, A., Brabant, P., Thiellement, H. and Alix, K. (2010) Analysis of gene expression in resynthesized Brassica napus allotetraploids: transcriptional changes do not explain differential protein regulation. New Phytol. 186, 216-227.

Ogawa, M., Kay, P., Wilson, S. and Swain, S.M. (2009) ARABIDOPSIS DEHISCENCE ZONE POLYGALACTURONASE1 (ADPG1), ADPG2, and QUARTET2 are Polygalacturonases required for cell separation during reproductive development in Arabidopsis. Plant Cell, 21, 216-233.

Østergaard, L. (2009) Don't 'leaf' now. The making of a fruit. Curr. Opin. Plant Biol. 12, 1-6.

Østergaard, L. and King, G.J. (2008) Standardized gene nomenclature for the Brassica genus. Plant Methods, 4, 10.

Østergaard, L., Kempin, S.A., Bies, D., Klee, H.J. and Yanofsky, M.F. (2006) Pod shatter-resistant Brassica fruit produced by ectopic expression of the FRUITFULL gene. Plant Biotechnol. J. 4, 45-51. 
Parkin, I.A.P., Gulden, S.M., Sharpe, A.G., Lukens, L., Trick, M., Osborn, T.C. and Lydiate, D.J. (2005) Segmental structure of the Brassica napus genome based on comparative analysis with Arabidopsis thaliana. Genetics, 171 765-781.

Petersen, M., Sander, L., Child, R., van Onckelen, H., Ulvskov, P. and Borkhardt, B. (1996) Isolation and characterisation of a pod dehiscence zone-specific polygalacturonase from Brassica napus. Plant Mol. Biol. 31, 517-527.

Pires, N. and Dolan, L. (2009) Origin and diversification of basic-helix-loophelix proteins in plants. Mol. Biol. Evol. 27, 862-874.

Price, J.S., Hobson, R.N., Neale, M.A. and Bruce, D.M. (1996) Seed losses in commercial harvesting of oilseed rape. J. Agric. Eng. Res. 80, 343-350.

Qiu, D., Morgan, C., Shi, J. et al. (2006) A comparative linkage map of oilseed rape and its use for QTL analysis of seed oil and erucic acid content. Theor. Appl. Genet. 114, 67-80.

Rajani, S. and Sundaresan, V. (2001) The Arabidopsis myc/bHLH gene ALCATRAZ enables cell separation in fruit dehiscence. Curr. Biol. 11, 19141922.

Riechmann, J.L., Krizek, B.A. and Meyerowitz, E.M. (1996) Dimerization specificity of Arabidopsis MADS domain homeotic proteins APETALA1, APETALA3, PISTILLATA and AGAMOUS. Proc. Natl Acad. Sci. USA, 93, 4793-4798.

Roberts, J.A., Elliott, K.A. and Gonzales-Carranza, Z.H. (2002) Abscission, dehiscence and other cell separation processes. Ann. Rev. Plant Biol. 53, 131-158.

Roeder, A.H., Ferrándiz, C. and Yanofsky, M.F. (2003) The role of the REPLUMLESS homeodomain protein in patterning the Arabidopsis fruit. Curr. Biol. 13, 1630-1635.

Rusholme, R.L., Higgins, E.E., Walsh, J.A. and Lydiate, D.J. (2007) Genetic control of broad-spectrum resistance to turnip mosaic virus in Brassica rapa (Chinese cabage). J. Gen. Virol. 88, 3177-3186.

Rutledge, R.G. and Côté, C. (2003) Mathematics of quantitative kinetic PCR and the application of standard curves. Nucleic Acids Res. 31, e93.
Sambrook, J. and Russell, D.W. (2001) Molecular Cloning. A laboratory manual. Cold Spring Harbor: Cold Spring Harbor Laboratory Press.

Smyth, D.R., Bowman, J.L. and Meyerowitz, E.M. (1990) Early flower development in Arabidopsis. Plant Cell, 2, 755-767.

Sorefan, K., Girin, T., Liljegren, S.J., Ljung, K., Robles, P., Galván-Ampudia C.S., Offringa, R., Friml, J., Yanofsky, M.F. and Østergaard, L. (2009) A regulated auxin minimum is required for seed dispersal in Arabidopsis. Nature, 459, 583-586.

Sparrow, P.A.C., Dale, P.J. and Irwin, J.A. (2004) The use of phenotypic markers to identify Brassica oleracea genotypes for routine high-throughput Agrobacterium-mediated transformation. Plant Cell Rep. 23, 64-70.

Sparrow, P.A.C., Dale, P.J. and Irwin, J.A. (2006) Brassica oleracea. In Meth ods in Molecular Biology, vol. 343: Agrobacterium protocols (Kan, W., ed). Totowa: Humana Press Inc, pp. 417-426.

Spence, J., Vercher, Y., Gates, P. and Harris, N. (1996) 'Pod shatter' in Arabidopsis thaliana, Brassica napus and B. juncea. J. Microscopy, 181, 195-203.

Stam, P. (1993) Construction of Integrated Genetic-Linkage Maps by Means of a New Computer Package - Joinmap. Plant J. 3, 739-744.

Stephenson, P., Baker, D., Girin, T., Perez, A., Amoah, S., King, G.J. and Østergaard, L.. (2010) A rich TILLING resource for studying gene function in Brassica rapa. BMC Plant Biol. 10, 62. doi:10.1186/1471-2229-10-62.

U. N. (1935) Genome analysis in Brassica with special reference to the experimental formation of $B$. napus and peculiar mode of fertilization. Jpn J. Bot. 7, 389-452.

Wang, N., Wang, Y., Tian, F., King, G.J., Zhang, C., Long, Y., Shi, L. and Meng, J. (2008) A functional genomics resource for Brassica napus: development of an EMS mutagenized population and discovery of FAE1 point mutations by TILLING. New Phytol. 180, 751-765.

Yang, Y.W., Lai, K.N., Tai, P.Y. and Li, W.H. (1999) Rates of nucleotide substitution in Angiosperm mitochondrial DNA sequences and dates of divergence between Brassica and other Angiosperm lineages. J. Mol. Evol. 48, 597-604.

GenBank accession number: GU386357 (BraA.IND.a) and GU386356 (BoIC.IND.a). 